Voix et Images

voixetimages

\title{
Le récit des femmes poètes
}

\section{Audrey Camus}

Volume 44, numéro 2 (131), hiver 2019

URI : https://id.erudit.org/iderudit/1059519ar

DOI : https://doi.org/10.7202/1059519ar

Aller au sommaire du numéro

Éditeur(s)

Université du Québec à Montréal

ISSN

0318-9201 (imprimé)

1705-933X (numérique)

Découvrir la revue

Citer ce compte rendu

Camus, A. (2019). Compte rendu de [Le récit des femmes poètes]. Voix et Images, 44(2), 113-117. https://doi.org/10.7202/1059519ar d'utilisation que vous pouvez consulter en ligne.

https://apropos.erudit.org/fr/usagers/politique-dutilisation/ 


\author{
R O M A N \\ Le récit des femmes poètes \\ $+++$
}

AUDREY CAMUS

Chercheuse indépendante

Le terme «roman» désignant pratiquement aujourd'hui par défaut toute narration fictionnelle, sans doute une chronique consacrée au genre ne peut-elle que se heurter régulièrement à la question de l'incertitude de ses limites. Autant donc s'y confronter sans délai, en évoquant pour commencer des ouvrages qui tendent à échapper à la dénomination.

Les villes de papier ${ }^{1}$ de Dominique Fortier, tout d'abord, s'emploie à relater l'existence d'Emily Dickinson. Considérée aujourd'hui comme une figure majeure de la littérature du XIXe siècle, la poétesse ne publia presque pas mais, en quasi-recluse, consacra toute son existence à l'écriture. Dominique Fortier, que les hasards de la vie conduisent à quitter Montréal pour la Nouvelle-Angleterre, lui emboîte le pas pour toucher du doigt le mystère de la création féminine et habiter, passagèrement, la "maison de ses poèmes» (152). Car si son livre se présente comme une sorte de biographie fictionnelle, $c^{\prime}$ est aussi un carnet d'écriture dans lequel l'auteure poursuit une réflexion sur la manière d'habiter le monde entamée avec Au péril de la mer². Aux «cabanes de mots» (33) qu'elle y évoquait succèdent ainsi les villes de papier, notamment celle qui porte le nom d'Emily, sur laquelle s'ouvre le récit. C'est cette ville «toute de bois blanc nichée au milieu de prairies de trèfles et d'avoine» (9) que Dominique Fortier va patiemment bâtir et nous amener à habiter avec elle tout au long du récit, en revisitant les épisodes de la vie de la poétesse qu'elle puise dans sa correspondance, emprunte à ses biographes ou construit de toutes pièces. Hardie comme une hérö̈ne de Jane Austen, la jeune Emily Dickinson se soustrait progressivement à toutes les obligations inhérentes à sa condition pour se livrer corps et âme à la poésie. Ses compagnes du séminaire étant chacune à son tour emportées par la mort ou par le mariage, Emily a compris qu'il lui faut choisir entre être femme et écrire. Elle va donc s'absenter au monde jusqu'à ne plus guère sortir du jardin, puis de la chambre, où elle compose ses poèmes étranges comme on épingle des papillons. S'il faut en croire Dominique Fortier, il serait vain de chercher un point de rupture susceptible d'expliquer cette réclusion. Dans Les villes de papier, on peut toutefois

1 Dominique Fortier, Les villes de papier, Québec, Alto, 2018, 187 p.

2 Dominique Fortier, Au péril de la mer, Québec, Alto, 2015, 171 p. 
penser que cette charnière symbolique coïncide avec le moment où Emily choisit de ne plus se vêtir que de blanc, car pour la romancière, «Emily Dickinson est un écran blanc, une page vierge. Eût-elle plutôt choisi, à la fin de sa vie, de passer une robe bleue, nous ne pourrions rien dire d'elle» (11). Ainsi son livre relate-t-il en quelque sorte l'effacement, la dématérialisation de cette femme qui a fait vœu de vivre dans une ville de papier (Linden, inventée par les cartographes pour déjouer les tentatives de récupération de leur travail). Mais il ne faudrait pas se méprendre pour autant; un tel effacement est d'une violence inouïe, ainsi que permet de le comprendre la scène clef où, dans la solitude de sa chambre, Emily se dépouille de tous les oripeaux de la vie sociale à mesure qu'elle se dénude: «[B]ientôt il ne lui restera plus qu'à sortir de sa peau et à se tenir devant son miroir, tout en dents et en côtes pointues, un petit squelette blanc comme neige.» (96) Emily n'est pas seulement inconvenante, elle est terrifiante. Telle que la dépeint Dominique Fortier, elle paraît tout entière tendue entre la grâce et l'ardeur. Car si la femme poète s'absente au monde, c'est pour mieux s'en emparer; pour faire entrer dans ses textes le miel du soleil et le tumulte des équinoxes comme jadis elle conservait les fleurs entre les pages des livres. Et si elle porte le blanc, c'est faute de pouvoir revêtir l'écarlate. Dans "ses poèmes qu'elle appelle neige», qu'on se garde bien de voir une dentelle délicate: "[E]n écrivant neige, ce qu'Emily a derrière les paupières, c'est la plus puissante des avalanches.» (144)

Sous la plume de Dominique Fortier, celle qui s'est dérobée à son destin tout tracé d'épouse et de mère afin de pouvoir écrire est peut-être essentiellement une femme pour qui « on ne peut pas avoir à la fois la vie et les livres - à moins de choisir les livres une fois pour toutes et d'y coucher sa vie» (108). À travers la figure d'Emily Dickinson, il est manifeste que l'écrivaine interroge son propre destin. Car, née un siècle et demi après son hérö̈ne, en un temps où la femme n'est plus confinée au foyer, elle a choisi le mariage et la maternité - et ses deux derniers livres qui entrelacent à la matière fictionnelle une sorte de journal d'écriture du déracinement font de son mari et de son enfant des personnages. Si la poétesse fournit à la "prosaïque» romancière la matière d'un livre, elle est aussi une sorte de prisme à travers lequel interroger la condition de femme vouée à la littérature, partagée entre le monde tangible et celui des livres, en diluant le «je» autobiographique dans l'encre du texte en train de s'écrire.

C'est aussi un destin de femme singulier que relate la poète Catherine Lalonde dans La dévoration des fées ${ }^{3}$, qui constitue une remarquable incursion en territoire narratif - mais un destin ancré dans la terre et la langue d'ici. Son héroïne, ensauvagée et sensuelle, semble au premier abord l'exact négatif de la diaphane Emily Dickinson, de la même manière que l'écriture viscérale de Catherine Lalonde et la prose délicate de Dominique Fortier paraissent s'opposer. Comme Les villes de papier, toutefois, ce récit est travaillé par la parole d'autres femmes poètes. L'auteure reconnaît ainsi sa dette à D. Kimm, Geneviève Desrosiers, Hélène Monette et Josée Yvon, et son

3 Catherine Lalonde, La dévoration des fées, Montréal, Le Quartanier, coll. «Série QR», 2017, 136 p. 
titre dit assez qu'il faut ajouter à cette liste le nom de Denise Boucher. Avec Les fées ont soif, cette dernière avait cherché à libérer la femme des archétypes de la mère, de la prostituée et de la Vierge auxquels la société l'assujettissait, en donnant voix aux personnages de Marie, de Madeleine et de la Statue. Quelque quarante ans plus tard, Catherine Lalonde reprend le flambeau dans un récit effervescent qui donne à lire l'émancipation féminine en mêlant au récit le conte, le mythe et la poésie. Le texte s'ouvre sur une naissance: celle de la p'tite, mise au monde dans la douleur par une mère qui ne lui survivra pas. C'est à Grand-Maman qu'il revient de prendre soin, à contrecœur, de cette enfant coupable d'avoir tué sa fille Blanche et de devoir connaître, comme toutes les autres, le triste sort des femmes (après les cris de l'enfantement, après le silence de la mort, après le vagissement du nouveau-né, c'est une exclamation de dépit qui retentit). Le texte raconte la grand-mère Carabosse aux prises avec la marmaille affamée qui toujours cavalcade. Dans l'absence de Blanche et des hommes - morts "comme des mouches» (55) - , Grand-Maman fulmine au milieu du désordre; « elle a hâte que la p'tite arrive, enfin, dans sa vie de femme faite; sa vie de femme faite de sang et d'eau de vaisselle» (53). Mais la p'tite va déjouer le sort. Malgré la mère morte et l'absence d'amour. Son premier mot sera non, et tout le reste à l'avenant. L'enfant préfère la fronde aux travaux d'aiguille et se soustrait aux corvées ménagères en braconnant. Adolescente, elle refuse les occupations de jeune fille pour s'abandonner à la force de son désir; «rêve de fuir aux lilas, aux garçons, aux framboises» (78). Et, à Grand-Maman qui attend qu'elle rentre dans le rang, elle répond en prenant le large... La frondeuse se livre alors à la ville, apprivoisant ses bruits et ses lumières pour, libre comme l'air, s'adonner au plaisir et enchanter les petits enfants. Ni mère, ni vierge, ni putain, songe-t-on, mais femme - cette femme à laquelle la pièce scandaleuse de Denise Boucher avait ouvert la voie. Puis, la p'tite va rentrer au bercail pour échapper une fois encore à la malédiction, par le don de la joie. C'est ainsi que l'héroïne de Catherine Lalonde, déjouant un à un les pièges tendus par l'existence en prenant les stéréotypes à rebours, répond finalement à l'appel de la Statue de Denise Boucher, qui demandait: «N'ai-je point quelque part une fille qui me délivrera, qui me déviergera ${ }^{4}$ ?» Rentrée de la ville, la p'tite devenue reine retourne à la sauvagerie originelle pour explorer cet en deçà du langage par lequel elle se prémunit contre l'interdit. Elle va délivrer Grand-Maman Carabosse du mauvais sort de sa vie de femme faite en lui offrant la jouissance perdue, dans une image qui trouve un double écho dans Les fées ont soif et dans le récit de Dominique Fortier donnant à voir Emily dénudée: «J'étais devenue statue dit Grand-Maman, j'étais squelette.» (126)

Ce faisant, la p'tite libère la parole poétique, le dit de l'aïeule qui, au seuil de la mort, conjugue la glace et le feu pour s'élever comme un chant de plénitude. Ainsi ce texte qui fait place au corps et aux cris - et au silence de l'absence - se referme-t-il sur un poème. Si Denise Boucher avait libéré ses fées en leur donnant la parole, Catherine Lalonde explore quant à elle la langue du corps pour offrir aux siennes l'assouvissement de la dévoration (le plaisir de rire, de manger et de jouir). Il ne s'agit plus pour l'auteure de dire le manque mais, en s'arc-boutant sur le discours

4 Denise Boucher, Les fées ont soif, Montréal, Éditions Intermède, 1979 [1978], p. 31. 
des femmes qui l'ont précédée (celui de Josée Yvon notamment), de le combler. Et dans le même temps, peut-on penser, de faire la généalogie de sa propre écriture.

Henri de ses décors ${ }^{5}$, enfin, par lequel Laurance Ouellet Tremblay pénètre à son tour en territoire fictionnel après avoir fait paraître plusieurs recueils de poèmes, résonne également de mots empruntés. Les influences dont le texte se réclame ne sont cependant plus uniquement féminines: Hervé Bouchard et Valère Novarina figurent ainsi, parmi d'autres, au côté de Sylvia Plath et de Denise Boucher (notons, en passant, qu'ailleurs l'auteure revendique elle aussi l'importance de Josée Yvon). Et la femme poète qui se consacre au récit prête ici sa voix à un homme. Mais, si ce questionnement dont procède le texte échappe au genre pour explorer la puissance de la parole et les rapports de force suscités par la création, c'est d'engendrement qu'il s'agit là encore. Croisement improbable du Bavard de Louis-René des Forêts et d'une créature insolite de Roland Topor, Henri est un personnage bien étrange: il prend le spectateur aux rets de son discours et finira, orgueil ou dérision, par se creuser la tête avec une cuillère à pamplemousse. Le texte s'ouvre sur le soliloque de ce personnage, et ne prendra fin que lorsqu'il se taira. Henri crée des décors de théâtre, en n'utilisant que du papier journal - journal qu'au demeurant il se refuse à lire. Il fait «tout, les ponts, les meubles, les ascenseurs et les bibelots, l'intérieur des matelas» (13), allant même jusqu'à concevoir, dans un étrange écho avec le livre de Dominique Fortier qui lui est contemporain, une ville de papier. Son travail suscite l'admiration, mais Henri semble préoccupé. Très vite, il interrompt son discours pour mettre les choses au point: «Je vous vois déjà prendre vos aises, déplier vos membres et vous installer confortablement sans avoir encore rien compris de notre entente; nous ne discuterons pas, voyez, c'est moi qui parlerai.» (15) Cette adresse vindicative donne d'emblée le ton du texte, en installant le lecteur dans la position du spectateur muet. Seul en scène, Henri se raconte, sans d'ailleurs pouvoir s'empêcher de prêter une opinion à l'interlocuteur qu'il a réduit au silence. Il raconte comment il est devenu créateur de décors, évoque ses parents, parle de ses relations avec «Olivier-le-chefdu-théâtre» (23) et avec Catherine, «la grande actrice» (57). Autant d'histoires qui, chacune à sa manière, renouent avec le romanesque. Mais ce sont d'abord les péripéties du discours que le texte relate. Henri ergote, cancane, ratiocine et, là comme lorsqu'il s'adresse au spectateur dont il a confisqué la parole, il n'est jamais question que de savoir qui exerce l'ascendant sur qui.

À l'entendre soliloquer de la sorte en faisant les questions et les réponses, on ne peut s'empêcher de songer au Bavard de Louis-René des Forêts ${ }^{6}$, qui pareillement donnait à lire le déploiement du discours et la confrontation au lecteur pour interroger les limites de la fiction. La filiation (que l'auteure prend soin de taire dans la reconnaissance des emprunts sur laquelle son livre se clôt) est d'autant plus forte qu'à l'instar du Bavard, le texte de Laurance Ouellet Tremblay se tisse d'autres textes.

5 Laurance Ouellet Tremblay, Henri de ses décors, Chicoutimi, La Peuplade, coll. «Récits», 2018, 72 p.

6 Louis-René des Forêts, Le Bavard, Paris, Gallimard, coll. «L'imaginaire», 1978 [1947], 168 p. 
Mais, là où Louis-René des Forêts faisait du discours le sujet de son livre, hypothéquant la fiction par la mise en cause du récit de son personnage qui revendiquait le mensonge, la femme poète déplace l'enjeu en donnant une scène à la voix, une portée au discours, un corps au personnage. Car contrairement au Bavard, qui n'avait de cesse de miner sa propre parole, Henri incarne la sienne (de ce point de vue, il n'est pas anodin qu'il s'adresse à des spectateurs plutôt qu'à des lecteurs). Henri n'est pas menteur, mais vaniteux. C'est un créateur, quand bien même ses créations sont des créations de papier: il confère aux mots des autres une réalité tangible. Ceux du texte dramatique, d'abord, auxquels il fournit un décor, mais aussi ceux de ses interlocuteurs, puisqu'il finira par obtempérer à l'injonction qui lui a été faite de se creuser la tête en s'entamant le crâne avec une cuillère à pamplemousse. Par cette monstrueuse relittéralisation d'une métaphore éculée, Henri fait basculer le texte dans une réalité étrange - la sienne - et, ce faisant, recharge le langage d'une efficace nouvelle: plutôt que l'exténuation du discours, c'est «l'engendrement par la parole ${ }^{7}$ » qui intéresse l'écrivaine.

$\bar{A}$ un correspondant qui lui demandait un jour comment elle se savait en présence de la poésie, Emily Dickinson répondit: "Quand je lis un livre qui me glace le corps à un point tel que nul feu ne saura jamais me réchauffer, je reconnais la poésie.» Puis: «Si j'ai l'impression qu'on m'arrache le crâne, je reconnais la poésie». (179) Cette définition que Dominique Fortier fait sienne en nous la transmettant, Catherine Lalonde et Laurance Ouellet Tremblay ne la désavoueraient sans doute pas. Par-delà leurs différences, ces trois textes laissent ainsi entendre de curieuses résonances. Pétris de poésie, ils font place à la voix et pourraient tous trois se lire à l'aune de la formule par laquelle l'auteure des Villes de papier caractérise le rapport au monde de son héroïne: «Chaque livre en contient cent autres. Ce sont des portes qui s'ouvrent et ne se referment jamais.» (44) Éminemment intertextuelle, l'hybridation du roman à laquelle les trois écrivaines se livrent leur permet de s'interroger sur leur propre pratique d'écriture et de la faire progresser sur le plan formel, en se réclamant d'une filiation qu'elles revendiquent pour mieux s'en émanciper. Dans la tension qu'ils instaurent entre texte et corps, le récit de soi pour Dominique Fortier, le poème pour Catherine Lalonde et le monologue thêâtral pour Laurance Ouellet Tremblay constituent aussi, plus profondément, autant de moyens de mettre à l'épreuve la puissance d'incarnation de la littérature.

7 C'est l'expression qui figure dans le titre de sa thèse: Laurance Ouellet Tremblay, "Ça ne finira pas, c'est parti pour l'éternité, fallait que vous le sachiez.» L'engendrement par la parole chez Hervé Bouchard, Pierre Perrault et Hector de Saint-Denys Garneau, thèse de doctorat, Montréal, Université du Québec à Montréal, 2016. 